\title{
高速主轴系统切削稳定性预测及影响因素分析“
}

\author{
汪 博 孙 伟 闻邦椿 \\ (东北大学机械工程与自动化学院 沈阳 110819)
}

\begin{abstract}
摘要: 高速主轴系统切削稳定性直接影响产品的表面加工精度和切削系统的使用寿命, 是评价高速主轴系统性能优劣的重要 因素, 而研究影响切削稳定性的因素并制定提高稳定性的合理途径同样也受到关注。提出从稳定性评价准则及系统频响函数 的不同求解方法入手, 分析无条件稳定区和有条件稳定区的影响因素, 总结出系统所受激励、主轴转速及系统结构是影响切 削稳定性的重要因素。以德国 GMN 高速主轴系统为例, 在采用 5 自由度轴承受力与变形关系模型模拟角接触球轴承和分布 式弹簧模型模拟主轴-刀柄-刀具结合部的基础上, 建立完整高速主轴系统通用有限元模型; 利用三维稳定性叶瓣图、极限切 削深度和叶瓣交点随参数的变化曲线表征切削力幅值、转速及阻尼比等参数对切削稳定性的影响规律, 为优化加工工艺、提 高系统切削稳定性提供理论依据。
\end{abstract}

关键词：高速主轴系统 主轴-刀柄-刀具结合部 切削稳定性 影响因素

中图分类号: TG502 TH113

\section{Stability Prediction and Influence Factors Analysis of High-speed Spindle System}

\author{
WANG Bo SUN Wei WEN Bangchun \\ (School of Mechanical Engineering \& Automation, Northeastern University, Shenyang 110819)
}

\begin{abstract}
The stability of high-speed spindle system affects the surface finish and the tool life directly, which is an important factor to evaluate the performance. It is concerned to research the influence factors and make a reasonable approach of improving the stability. Starting from the stability evaluation criteria and different ways of getting FRF, the influence factors of unconditional and conditional stability regions including motivation, speed and spindle system structure are analyzed. Taking GMN spindle as sample, the whole high-speed spindle system finite element method is modeled with five degrees model of angular contact ball bearing taking the force and deformation in consideration and distributing spring model of spindle - holder - tool joints. The influence laws of cutting force amplitude, speed and damping ratio towards cutting stability are characterized by the three dimensional lobes, limit cutting depths and lobe intersections changing with the parameters, which provide the theoretical basis for optimizing the processing and improving the cutting stability.
\end{abstract}

Key words: High-speed spindle Spindle-holder-tool joints Cutting stability Influence factor

\section{0 前言}

机床或加工中心抵抗切削过程振动(包括受迫 振动和自激振动等)的能力, 称为切削稳定性, 也称 机床抗振性。机床的㗜振起源于加工过程中切屑形 成过程中的自激机理, 对工件的去除率、加工精度、 生产效率以及机床和刀具的使用寿命都具有极大的

*中央高校基本科研业务费专项资金(N110603004)、国家自然科学基金 (50905029)和辽宁省教育厅科学研究一般项目(L2013115)资助项目。 20130407 收到初稿, 20130709 收到修改稿
影响。在高速加工中心广泛应用于汽车、模具、航 空航天等精密制造业的今天, 切削稳定性的研究越 来越受到重视。

INSPERGER 等 ${ }^{[1]}$ 应用半离散化方法研究了单 自由度铣削系统的稳定性。SMITH 等 ${ }^{[2]}$ 提出了时域 仿真的方法构建铣削颤振过程的稳定性 Lobe 图。 SCHMITZ $^{[3]}$ 、AHMADI 等 ${ }^{[4]}$ 利用子结构法预测高速 加工中心的动态特性及切削稳定性。山东大学唐委 校 $^{[5]}$ 给出了高切削速度情况下多自由度系统高阶固 有频率影响的切削稳定性极限判据及其预测分析 方法。

随着研究的深入, 学者们开始对切削稳定性的 
影响因素展开探索, 主要围绕着刀具长度、刀具类 型以及主轴速度等方面。TLUSTY ${ }^{[6]}$ 讨论了主轴配 有长端铣刀的常规模态, 以及端铣刀长度的变化在 不同转速下对切削稳定性的影响。SMITH 等 ${ }^{[7]}$ 和 DAVIES 等 ${ }^{[8]}$ 分别针对细长立铣刀的高速铣削稳定 性, 低径向切深铣削的稳定性, 以及内圆高速铣削 振动对工件质量的动态影响等问题进行了研究。

ISMAIL 等 ${ }^{[9]} 、 \mathrm{JENSEN}^{[10]}$ 和 ALTINTAS 等 ${ }^{[11]}$ 分别对 不等间距刀具、球头立铣刀和面铣刀的切削稳定性 进行分析预测。SCHMITZ 等 ${ }^{[12]}$ 提出非旋转和旋转 刀具频响函数 FRF 测试方法。宋清华 ${ }^{[13]}$ 研究刀具主 要结构参数对铣削系统稳定性的影响规律。

现代制造业对切削系统稳定性的要求不断提 高, 曾经被忽略或简化的影响因素重新受到关注, 成为提高系统切削稳定性的关键。为此, 本文从稳 定性评价准则入手, 提出了除无条件稳定区域之外, 有条件稳定区域同样是影响切削稳定性的关键; 并 在深入分析极限切削深度和系统频响函数的不同求 解方法之后, 总结出系统所受的激励、转速、阻尼 比及主轴-刀柄-刀具结合部都会对切削稳定性产生 影响。以 GMN 高速主轴系统为例, 采用分布式弹 簧模型模拟主轴-刀柄-刀具结合部, 建立完整的高 速主轴系统有限元模型, 利用三维稳定性叶瓣图、 极限切削深度和叶瓣交点随参数的变化曲线表征切 削力、转速及阻尼比等参数对切削稳定性的影响规 律, 对实际切削加工中的稳定性提高具有指导性 意义。

\section{1 切削稳定性影响因素}

评价切削系统稳定性通常是通过稳定性叶瓣 图, 其中极限切削深度是表征切削稳定性的重要指 标, 如式(1)所示 ${ }^{[14]}$

$$
b_{\lim }=\frac{-1}{2 \times Z K_{f} G\left(\omega_{c}\right)}
$$

式中, $b_{\lim }$ 为无颤振最大轴向切削深度, $Z$ 为刀具齿 数, $K_{f}$ 为进给方向切削力系数, $G\left(\omega_{c}\right)$ 为频响函数的 实部值, $\omega_{c}$ 为使频响函数实部为负的角频率。

在叶瓣图中 $b_{\mathrm{lim}}$ 以下的区域是无条件稳定区 域, $b_{\lim }$ 的值越大, 切削系统越稳定, $b_{\lim }$ 的影响因 素包括刀具齿数 $Z$, 切削力系数 $K_{f}$ 和系统频响函数 实部 $G$ 。如果无条件稳定区不变, 在保证稳定切削 前提下使用更大的切削深度, 于是, $b_{\mathrm{lim}}$ 值以上, 叶瓣交点(即稳定区域与不稳定区域的临界点)以下 的有条件稳定区域成为研究的重点。也就是说, 在 一定的主轴工作转速下, 即使选择了高于 $b_{\lim }$ 值的
切削深度, 加工过程也存在稳定的可能, 这也是有 条件稳定区的意义。有条件稳定区的分布受叶瓣形 状及位置的影响, 而叶瓣形状及位置同样受系统频 响函数实部的影响。因此, 系统频响函数成为影响 切削稳定性的关键因素之一。

系统频响函数除了可以通过试验模态测试的 方法获得之外, 还可以通过直接法或固有特性法等 分析计算方法获得。

所谓直接法就是利用频响函数的概念直接求 解的方法。在线性定常系统中, 当初始条件为零时, 系统的响应(或输出)与激励(或输入)的拉普拉斯变 换之比, 如式(2)所示

$$
\phi(s)=\frac{y(s)}{F(s)}
$$

固有特性法是从频响函数的本质入手, 利用系 统的固有特性获得系统频响函数的方法。当系统的 运动方程为 $\boldsymbol{m} \ddot{\boldsymbol{x}}+\boldsymbol{c} \dot{\boldsymbol{x}}+\boldsymbol{k} \boldsymbol{x}=\boldsymbol{F}(t)$ 时, 对两端进行拉氏 变换得 $\left(\boldsymbol{m} s^{2}+\boldsymbol{c} s+\boldsymbol{k}\right) \overline{\boldsymbol{x}}(s)=\overline{\boldsymbol{F}}(s)$, 于是有

$$
\boldsymbol{H}_{d}(s)=\frac{\overline{\boldsymbol{x}}(s)}{\overline{\boldsymbol{F}}(s)}=\left(\boldsymbol{m} s^{2}+\boldsymbol{c} s+\boldsymbol{k}\right)^{-1}
$$

式中, $\boldsymbol{H}_{d}(s)$ 为系统的传递函数矩阵, $\overline{\boldsymbol{F}}(s)$ 为系统激 励矢量的拉氏变换, $\overline{\boldsymbol{x}}(s)$ 为系统响应矢量的拉氏变 换, $\boldsymbol{m} 、 \boldsymbol{k} 、 \boldsymbol{c}$ 为系统质量、刚度、阻尼矩阵。由于 矩阵的正交性可以推导出

$$
\left\{\begin{array}{l}
\boldsymbol{m}=\left(\boldsymbol{\Phi}^{\mathrm{T}}\right)^{-1} \boldsymbol{\Phi}^{-1} \\
\boldsymbol{c}=\left(\boldsymbol{\Phi}^{\mathrm{T}}\right)^{-1} \operatorname{diag}\left(2 \xi_{i} \omega_{i}\right) \boldsymbol{\Phi}^{-1} \\
\boldsymbol{k}=\left(\boldsymbol{\Phi}^{\mathrm{T}}\right)^{-1} \operatorname{diag}\left(\omega_{i}^{2}\right) \boldsymbol{\Phi}^{-1}
\end{array}\right.
$$

将式(4)代入式(3)中, 令 $s=\mathrm{j} \omega$, 则 $q$ 点激励、 $p$ 点拾取响应的位移频响函数为

$$
H_{d}^{p q}(\omega)=\sum_{i=1}^{n} \frac{\phi_{p i} \phi_{q i}}{\omega_{i}^{2}-\omega^{2}+\mathrm{j} 2 \xi_{i} \omega_{i} \omega}
$$

式中, $\phi_{p i} 、 \phi_{q i}$ 分别为 $p$ 点和 $q$ 点的第 $i$ 阶振型, $\zeta_{i}$ 为系统第 $i$ 阶阻尼比, $\omega_{i}$ 为系统第 $i$ 阶固有角频率, $\omega$ 为系统所受激振角频率。

从式(2)、(3)、(5)中可以看出, 影响系统频响 函数的因素众多，包括系统所受外界激励 $F$ 、系统 响应 $y$ 、系统结构参数中质量 $m$ 、刚度 $k$ 、阻尼 $c$ 及 其影响的振型 $\phi$ 、固有角频率 $\omega$ 和阻尼比 $\zeta$ 等, 另 外主轴-刀柄-刀具结合部对系统动力学特性的影响 也已经被证实 ${ }^{[15]}$, 而速度引起的陀螺力矩和离心力 对系统频响函数的影响也越来越大, 因此转速对系 统切削稳定性的影响不容忽视 ${ }^{[16]}$, 如图 1 所示。而 本文重点研究切削力、转速、阻尼比等因素对切削 稳定性的影响, 如图 2 所示。 


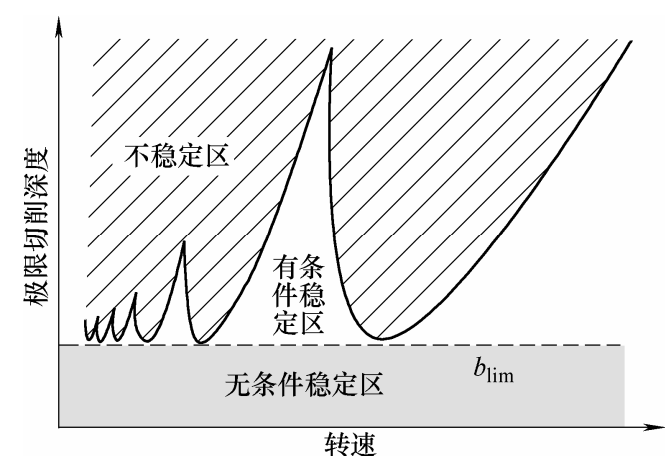

图 1 极限稳定性叶瓣图

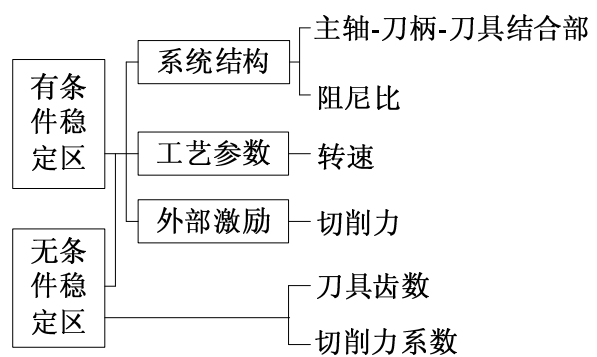

图 2 切削稳定性影响因素

\section{2 有限元模型和稳定性的预测方法}

\section{1 建立高速主轴系统有限元模型}

选用 GMN 公司的 HCS150 型电主轴, 安装 BB-T50-MEGA32D-165 刀柄以及 HLXX32 削平直 柄立铣刀。选用等参梁单元建立高速主轴系统的模 型, 系统运行时存在轴向平动和转动分量, 因此每 个单元在每个节点上有 3 个平动自由度和 3 个转动 自由度, 每个单元共 12 个自由度, 分别用 $q_{1} \sim q_{12}$ 表 示, 包括材料内阻的梁单元运动微分方程可表示

$$
\boldsymbol{m}_{e} \ddot{\boldsymbol{q}}_{e}+\left(\alpha \boldsymbol{m}_{e}+\beta \boldsymbol{k}_{e}+n_{\Omega} \boldsymbol{J}_{e}\right) \dot{\boldsymbol{q}}_{e}+\left(\boldsymbol{k}_{e}+\boldsymbol{k}_{e, c}\right) \boldsymbol{q}_{e}=\boldsymbol{Q}_{e} \text { (6) }
$$

式中, $\boldsymbol{m}_{e} 、 \boldsymbol{J}_{e} 、 \boldsymbol{k}_{e}$ 分别为单元质量矩阵、单元陀螺 效应矩阵、单元刚度矩阵, $\boldsymbol{k}_{e, c}$ 为旋转产生的离心刚 度矩阵, $\boldsymbol{Q}_{e}$ 为单元外力矢量, $\boldsymbol{q}_{e}$ 为单元节点位移矢 量, $\alpha 、 \beta$ 为比例阻尼常数, $n_{\Omega}$ 为扫频转速。

高速主轴单元中的角接触球轴承采用 5 自由度 接触受力模型, 考虑所有滚动体对轴承内滚道的作 用力，建立轴承内滚道的受力平衡方程为

$$
\begin{gathered}
F_{x}-\sum_{j=1}^{N_{b}} Q_{\mathrm{ij}} \sin \alpha_{\mathrm{i} j}=0 \\
F_{y}-\sum_{j=1}^{N_{b}} Q_{\mathrm{ij}} \cos \alpha_{\mathrm{i} j} \cos \psi_{j}=0 \\
F_{z}-\sum_{j=1}^{N_{b}} Q_{\mathrm{ij}} \cos \alpha_{\mathrm{i} j} \sin \psi_{j}=0 \\
M_{y}-\sum_{j=1}^{N_{b}} Q_{\mathrm{ij}} \cos \alpha_{\mathrm{ij}} R_{\mathrm{i}} \sin \psi_{j}=0 \\
M_{z}-\sum_{j=1}^{N_{b}} Q_{\mathrm{ij}} \sin \alpha_{\mathrm{ij}} R_{\mathrm{i}} \cos \psi_{j}=0
\end{gathered}
$$

式中, $F_{x} 、 F_{y} 、 F_{z}$ 分别为主轴对轴承内滚道作用的 轴向力和径向力, $M_{y} 、 M_{z}$ 为力矩载荷, $Q_{\mathrm{ij}}$ 为第 $j$ 个滚珠在内滚道所受法向载荷, $\alpha_{\mathrm{i} j}$ 为第 $j$ 个滚珠在 承载状态下的接触角, $\psi_{j}$ 为第 $j$ 个滚珠的位置角, $R_{\mathrm{i}}$ 为内滚道曲率中心的半径, $N_{b}$ 为轴承内滚珠个数。

将每个单元的质量、刚度、阻尼矩阵进行组集, 考虑角接触球轴承的轴承力、主轴-刀柄-刀具结合 部的建模方法 ${ }^{[15]}$, 形成整个高速主轴系统的动力学 方程为

$$
\begin{gathered}
\boldsymbol{m} \ddot{\boldsymbol{X}}+\boldsymbol{c} \dot{\boldsymbol{X}}+\boldsymbol{k} \boldsymbol{X}=\boldsymbol{F}_{R}(\boldsymbol{X})+\boldsymbol{F}_{H}(\boldsymbol{X}, \dot{\boldsymbol{X}})+\boldsymbol{F}_{P}(t)+\boldsymbol{G} \\
\boldsymbol{c}=\boldsymbol{D}+n_{\Omega} \boldsymbol{J}+\boldsymbol{k}_{H} \\
\boldsymbol{k}=\boldsymbol{k}_{s}+\boldsymbol{k}_{C} \\
\boldsymbol{X}=\left(\boldsymbol{q}_{1}, \boldsymbol{q}_{2}, \cdots, \boldsymbol{q}_{53}\right)^{\mathrm{T}} \\
\boldsymbol{q}_{i}=\left(x_{i}, y_{i}, z_{i}, \theta_{x i}, \theta_{y i}, \theta_{z i}\right)^{\mathrm{T}} \quad i=1,2, \cdots, 53
\end{gathered}
$$

式中, $\boldsymbol{D}$ 为材料阻尼矩阵, $\boldsymbol{J}$ 为系统的陀螺力矩阵, $\boldsymbol{k}_{H}$ 为结合部耦合刚度矩阵, $\boldsymbol{k}_{s}$ 为主轴刚度矩阵, $\boldsymbol{k}_{C}$ 为主轴离心刚度矩阵, $\boldsymbol{F}_{R}$ 为轴承支承力矢量, $\boldsymbol{F}_{H}$ 为结合部滞回力矢量, $\boldsymbol{F}_{P}$ 为系统切削力矢量, $\boldsymbol{G}$ 为 系统所受重力矢量, $\boldsymbol{X}$ 为系统位移矩阵, $\boldsymbol{q}_{i}$ 为节点 $i$ 处的位移矢量, $x_{i} 、 y_{i} 、 z_{i} 、 \theta_{x i} 、 \theta_{y i} 、 \theta_{z i}$ 为节点 $i$ 的 3 个位移和 3 个转角。

图 3 所示为高速主轴系统经过离散后的多自由 度有限元模型，圆点表示质点，每一个小段表示一 个单元，每个单元有 2 个节点组成，整个系统共有 52 个单元和 55 个节点, 其中包括 2 个过渡单元。 电主轴、刀柄、刀具的具体尺寸如表 1 3 所示。

相关动力学参数默认值如下: 弹性模量 $E=2.06 \times 10^{11} \mathrm{~Pa}$, 材料密度 $\rho=7850 \mathrm{~kg} / \mathrm{m}^{3}$, 主轴-刀 柄结合部接触刚度 $k_{s h}=1 \times 10^{8} \mathrm{~N} / \mathrm{m}$, 刀柄-刀具结合 部接触刚度 $k_{h l}=1 \times 10^{8} \mathrm{~N} / \mathrm{m}$, 轴承弹簧刚度为 $k_{b}=1 \times 10^{8} \mathrm{~N} / \mathrm{m}$, 刀具齿数 $Z=4$, 默认扫频转速 $n_{\Omega}=$ $20000 \mathrm{r} / \mathrm{min}$, 切削力系数 $K_{f}=2600 \mathrm{~N} / \mathrm{mm}^{2}$ 。其中结 合部刚度的数量级是通过试验辨识得到的, 而轴承 刚度的数量级是通过分析滚珠轴承特性得到的。

\section{2 稳定性预测方法}

在完成高速主轴系统有限元模型并求解系统 频响函数的基础上, 绘制叶瓣图, 进而预测高速主 轴系统切削稳定性, 具体步骤如下。

(1) 将系统传递函数分解成实部和虚部形式 $\phi\left(\mathrm{j} \omega_{c}\right)=G+\mathrm{j} H$ 。只有在传递函数实部 $G\left(\omega_{c}\right)$ 为负值时 切削深度才有物理意义, 因此求解所有满足 $G\left(\omega_{c}\right)$ 
$<0$ 的激振角频率 $\omega_{c}$ 的值。根据所加工材料选取 $K_{f}$ 的值, 并根据式(1)计算 $b_{\text {lim }}$ 。

(2) 判断在所选取的 $\omega_{c}$ 范围内, 传递函数的虚 部 $H\left(\omega_{c}\right)$ 的正负。如果 $H\left(\omega_{c}\right)<0$ 那么系统的相位角 $\psi=-\pi+\arctan (H / G)$; 如果 $H\left(\omega_{c}\right)>0$ 那么系统的相位
角 $\psi=-\pi-\arctan (|H / G|)$ 。根据 $\varepsilon=3 \pi+2 \psi$ 求得内外波纹 之间的相位差 $\varepsilon 、 T=(1+\varepsilon / 2 \pi) / f_{c}$ 及主轴转速 $n=60 / T$ 。

(3) 设定所绘制稳定性叶瓣图中叶瓣个数 $l$, 在 每个叶瓣内计算 $b_{\lim }$ 和 $n$, 将 $l$ 个循环周期绘制在同 一张图上, 就形成了极限稳定性叶瓣图。

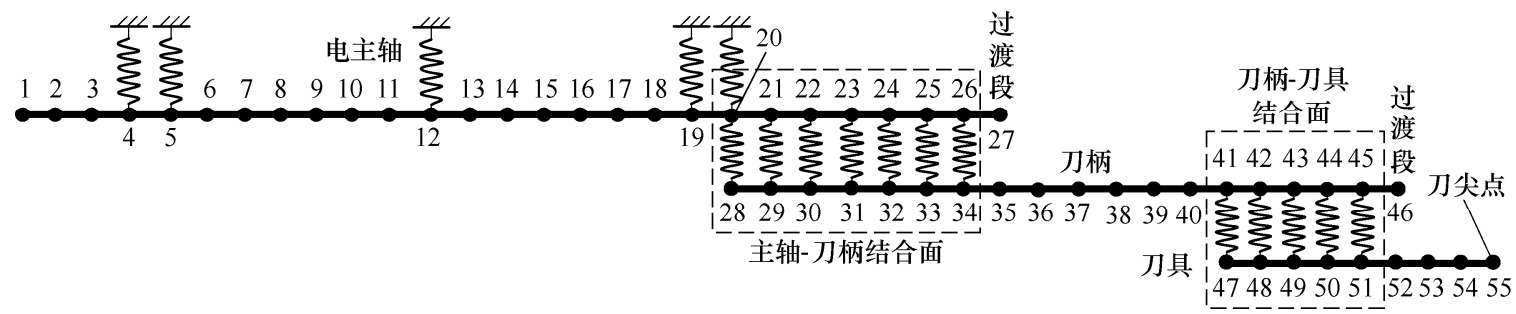

图 3 高速主轴系统有限元模型

表 1 电主轴尺寸

\begin{tabular}{cccc||cccc||cccc}
\hline 轴段 & 长度 & 外径 & 内径 & 轴段 & 长度 & 外径 & 内径 & 轴段 & 长度 & 外径 & 内径 \\
\hline 1 & 14.5 & 120.0 & 70.0 & 10 & 30.0 & 150.0 & 65.0 & 19 & 15.0 & 150.0 & 55.0 \\
2 & 25.0 & 149.9 & 70.0 & 11 & 15.0 & 150.0 & 55.0 & 20 & 22.6 & 149.9 & 40.2 \\
3 & 15.0 & 149.9 & 55.0 & 12 & 30.0 & 150.0 & 65.0 & 21 & 22.6 & 129.9 & 46.8 \\
4 & 15.0 & 149.9 & 55.0 & 13 & 30.0 & 150.0 & 65.0 & 22 & 22.6 & 129.9 & 53.4 \\
5 & 25.0 & 149.9 & 65.0 & 14 & 30.0 & 150.0 & 55.0 & 23 & 22.6 & 129.9 & 60.0 \\
6 & 30.0 & 149.9 & 65.0 & 15 & 25.0 & 150.0 & 55.0 & 24 & 11.4 & 88.0 & 66.6 \\
7 & 25.0 & 149.9 & 65.0 & 16 & 25.0 & 150.0 & 55.0 & 25 & 5.0 & 88.0 & 69.9 \\
8 & 25.0 & 149.9 & 65.0 & 17 & 25.0 & 150.0 & 70.0 & & & & \\
9 & 30.0 & 150.0 & 65.0 & 18 & 15.0 & 150.0 & 55.0 & & & & \\
\hline
\end{tabular}

表 2 刀柄尺寸

\begin{tabular}{cccc||cccc}
\hline 轴段 & 长度 & 外径 & 内径 & 轴段 & 长度 & 外径 & 内径 \\
\hline 1 & 22.6 & 40.2 & 25.0 & 10 & 12.0 & 95.0 & 20.0 \\
2 & 22.6 & 46.8 & 24.0 & 11 & 12.0 & 90.0 & 20.0 \\
3 & 22.6 & 53.4 & 24.0 & 12 & 10.0 & 86.0 & 20.0 \\
4 & 22.6 & 60.0 & 20.0 & 13 & 22.0 & 86.0 & 32.0 \\
5 & 11.4 & 66.6 & 20.0 & 14 & 20.0 & 86.0 & 32.0 \\
6 & 5.0 & 69.9 & 20.0 & 15 & 20.0 & 86.0 & 32.0 \\
7 & 19.0 & 100.0 & 20.0 & 16 & 20.0 & 86.0 & 32.0 \\
8 & 7.4 & 85.0 & 20.0 & 17 & 11.0 & 80.0 & 32.0 \\
9 & 11.4 & 100.0 & 20.0 & & & & \\
\hline
\end{tabular}

\begin{tabular}{cccc||cccc} 
& & & \multicolumn{2}{c}{ 表 3 } & \multicolumn{2}{c}{ 立铣刀尺寸 } & \multicolumn{2}{c}{$\mathrm{mm}$} \\
\hline 轴段 & 长度 & 外径 & 内径 & 轴段 & 长度 & 外径 & 内径 \\
\hline 1 & 20.0 & 32.0 & 0 & 5 & 8.0 & 30.0 & 0 \\
2 & 20.0 & 32.0 & 0 & 6 & 18.0 & 25.6 & 0 \\
3 & 20.0 & 32.0 & 0 & 7 & 18.0 & 25.6 & 0 \\
4 & 11.0 & 32.0 & 0 & 8 & 20.0 & 25.6 & 0 \\
\hline
\end{tabular}

3 切削稳定性的影响规律

\section{1 切削力对系统稳定性的影响}

采用简谐激振力模拟高速主轴系统切削过程 中的切削力, 通过改变简谐激振力的幅值来分析切 削力大小对高速主轴系统切削稳定性的影响规律。
图 4 为两种不同切削力时系统极限稳定叶瓣图, 从 图中可以看出, 切削力增大直接导致极限切削深度 的降低, 并且对有条件稳定区也产生影响。

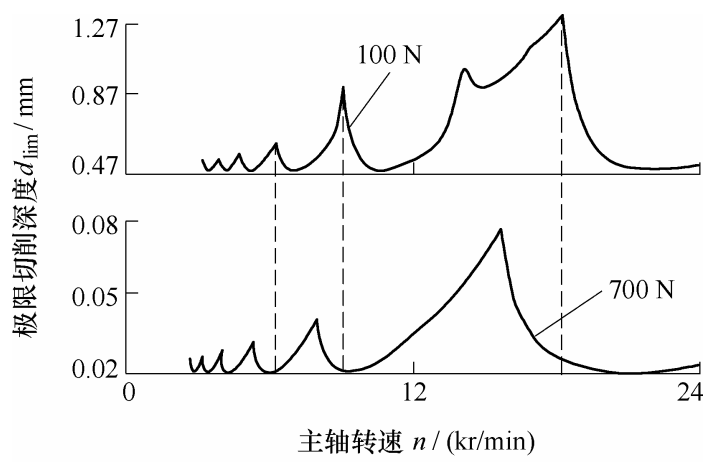

图 4 不同切削力下系统稳定叶瓣图

图 5a 为不同切削力下系统切削三维稳定性叶 瓣图, 图 $5 \mathrm{~b}$ 为最小极限切削深度随切削力的变化曲 线, 图 $5 \mathrm{c}$ 为叶瓣交点所对应临界转速随切削力的变 化曲线。从图 $5 \mathrm{a}$ 和图 $5 \mathrm{~b}$ 中可以看出, 切削力增大, 极限切削深度在不断降低, 在切削力较小时, 极限 切削深度受切削力影响较大, 而当切削力较大时, 极限切削深度变化趋势接近平缓; 图 5c 所示一阶叶 瓣交点是 $l=0$ 和 $l=1$ 的交点, 二阶叶瓣交点是 $l=1$ 和 $l=2$ 的交点, 研究发现稳定与不稳定区域的临界 点(即叶瓣交点)随切削力增大向左发生移动。 


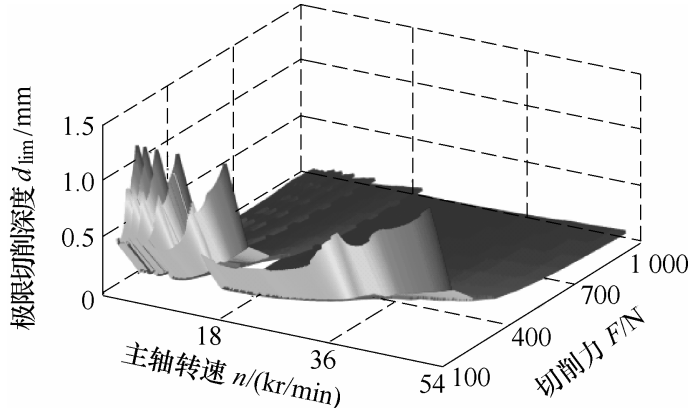

(a) 三维稳定性叶瓣图

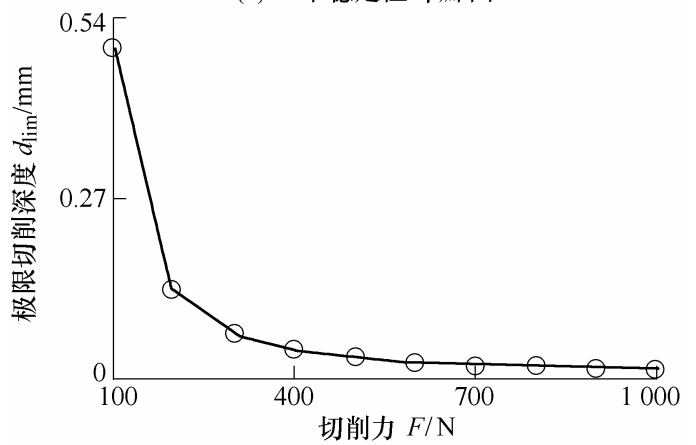

(b) 最小极限切削的变化

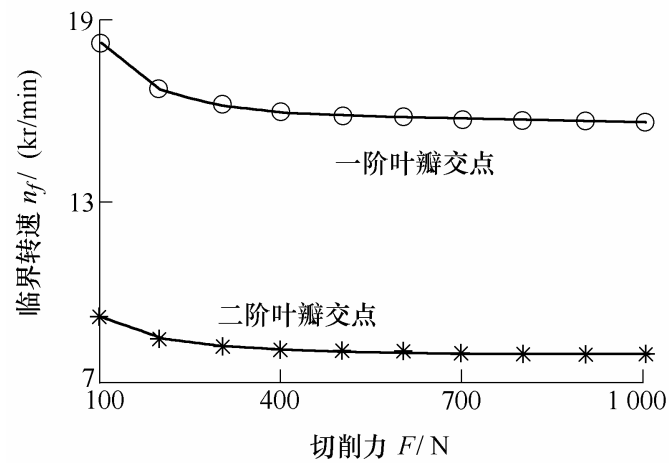

(c) 叶瓣交点的变化

图 5 不同切削力对切削稳定性的影响

\section{2 转速对系统稳定性的影响}

图 6a 所示为在不同转速下高速主轴系统切削 稳定性三维谱图, 图 $6 \mathrm{~b}$ 为最小极限切削深度随转速 的变化曲线, 图 $6 \mathrm{c}$ 为叶瓣交点所对应临界转速随转 速的变化曲线。从图 6a 中可以看出, 随着主轴转速 $n_{\Omega}$ 的升高, 系统的极限稳定性叶瓣图曲线在逐渐升 高, 说明系统的切削稳定性随着主轴转速的升高而 增强。图 $6 \mathrm{~b}$ 中转速对系统一阶固有频率所对应的最 小极限切削深度影响较大, 而对二阶固有频率所对 应的最小极限切削深度影响较小。从图 $6 \mathrm{c}$ 中可以看 出, 随着 $n_{\Omega}$ 的增大, 叶瓣交点向右移动, 这说明随 着扫频转速的增大, 系统有条件稳定区域发生改变。

\section{3 阻尼系数对系统稳定性的影响}

图 7a 为不同阻尼系数时切削稳定性三维图谱, 图 $7 \mathrm{~b}$ 为最小极限切削深度随阻尼系数的变化曲线, 图 7c 为叶瓣交点随阻尼系数的变化曲线。从图 $7 \mathrm{a}$ 中可以看出, 当阻尼系数从 $1 \times 10^{-5}$ 到 $1 \times 10^{-2}$ 范围内 变化时, 系统的切削稳定性是逐渐增强的。图 $7 \mathrm{~b}$

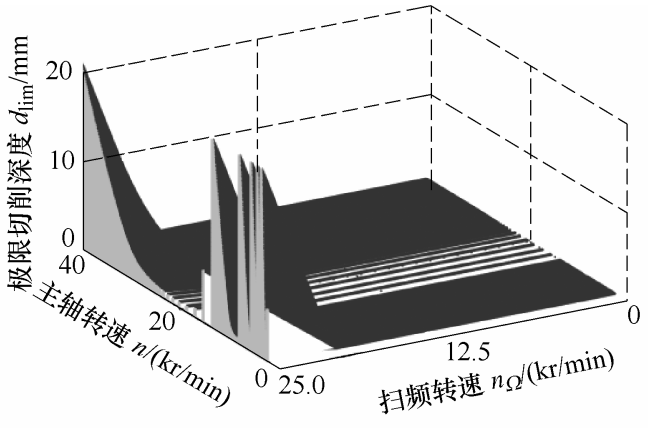

(a) 三维稳定性叶瓣图

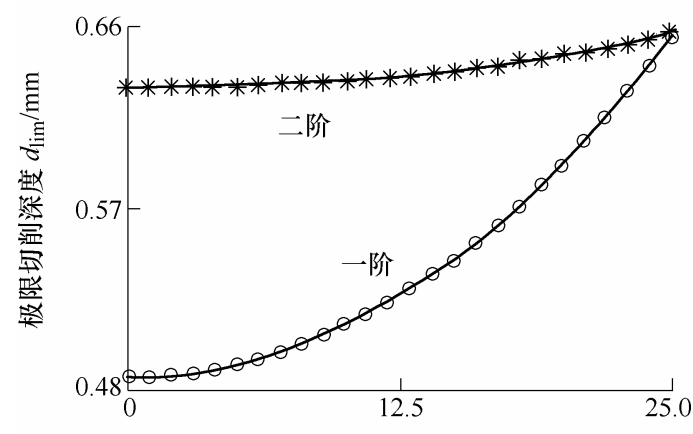

扫频转速 $n_{\Omega} /(\mathrm{kr} / \mathrm{min})$

(b) 最小极限切削的变化

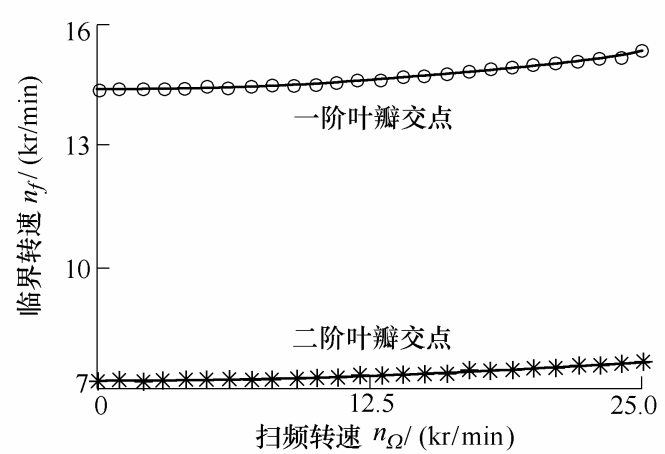

(c) 叶瓣交点的变化

图 6 不同主轴转速对切削稳定性的影响

中, 当阻尼系数很小 $\left(\xi<1 \times 10^{-3}\right)$ 时, 高速主轴系统最 小极限切削深度随阻尼系数的变化幅度很小, 当阻 尼系数增大 $\left(\xi>1 \times 10^{-3}\right)$ 后, 高速主轴系统最小极限切 削深度随阻尼系数变化幅度增大。从图 $7 \mathrm{c}$ 中可以看 出, 稳定性临界点受阻尼系数影响很小。

\section{4 结论}

(1) 提出了研究稳定性叶瓣图中无条件稳定区 和有条件稳定区对提高切削系统稳定性同样重要的 观点, 并根据系统频响函数的不同求解方法总结出 切削力、转速、阻尼比和主轴-刀柄-刀具结合部是 影响切削稳定性的关键因素。

(2) 在采用 5 自由度轴承受力与变形关系模型 模拟角接触球轴承, 分布式弹簧模拟主轴-刀柄-刀 具结合部的基础上建立了完整高速主轴系统通用有 限元模型。 


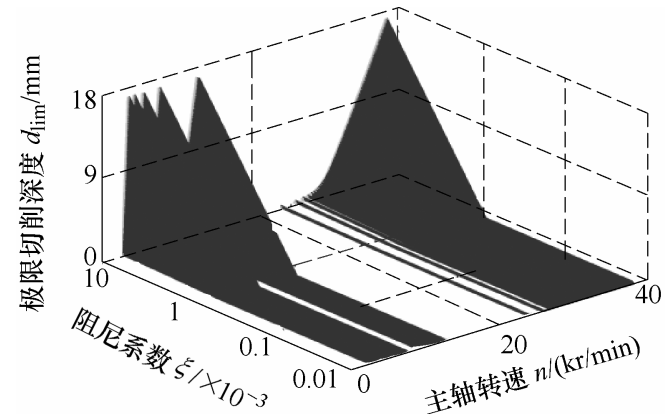

(a) 三维稳定性叶瓣图

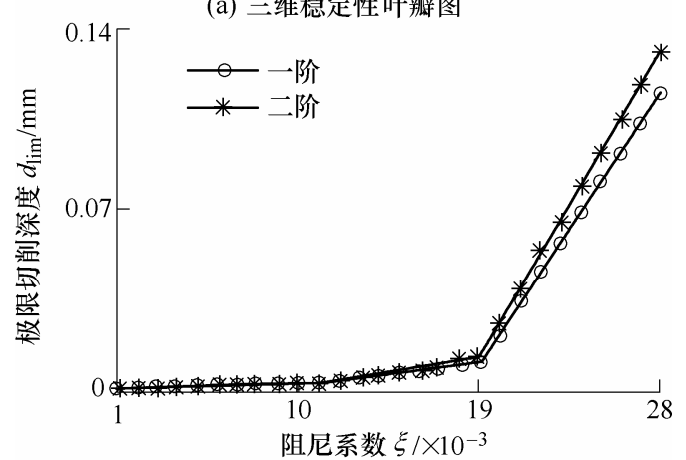

(b) 最小极限切削的变化

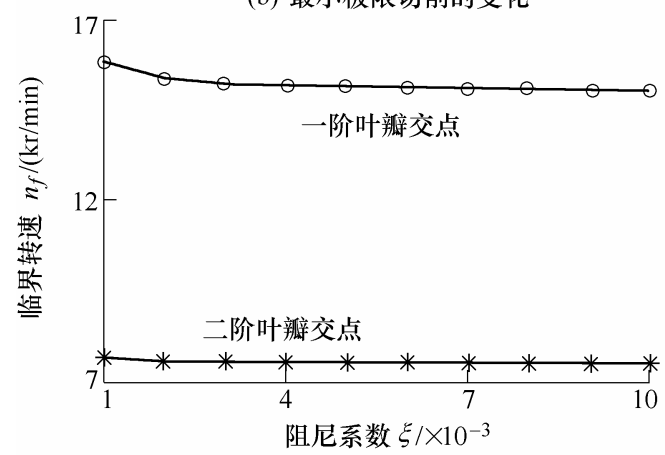

(c) 叶瓣交点的变化

图 7 不同阻尼系数对切削稳定性的影响

(3) 根据分析所得, 大切削力使得高速主轴系 统切削稳定性下降; 转速增大使得系统无条件稳定 区扩大，但有条件稳定区发生右移; 阻尼系数的增 大, 使得系统稳定性增强。因此, 在制定工件的加 工工艺时, 可以在允许的范围内降低切削力并增大 系统阻尼系数, 而主轴转速对系统稳定性影响不是 简单的对应关系, 需要具体转速具体分析。

\section{参 考 文 献}

[1] INSPERGER T, MANN B P, STÉPÁN G, et al. Stability of up-milling and down-milling, part 1: Alternative analytical methods[J]. International Journal of Machine Tools \& Manufacture, 2003(43): 25-34.

[2] SMITH S, TLUSTY J. Efficient simulation programs for chatter in milling [J]. Annals of the CIRP-Manufacturing Technology, 1993, 42(1): 463-466.

[3] SCHMITZ T L. Predicting high-speed machining dynamics by substructure analysis[J]. Annals of the
CIRP, 2000, 49(1): 303-308.

[4] AHMADI K, AHMADIAN H. Modeling machine tool dynamics using a distributed parameter tool-holder joint interface $[\mathrm{J}]$. International Journal of Machine Tools \& Manufacture, 2007, 47: 1916-1928.

[5] 唐委校. 高速切削稳定性及其动态优化研究[D]. 济南: 山东大学, 2005.

TANG Weixiao. Research on high-speed machining stability and dynamic optimization[D]. Jinan: Shandong University, 2005.

[6] TLUSTY J. Dynamics of high-speed milling [J]. Journal of Engineering for Industry, 1986, 108: 59-67.

[7] SMITH S, WINFOUGH W R, PRATT J R, et al. The effect of tool length on stable metal removal rate in high-speed milling[J]. Annals of the CIRP, 1998, 47(1): 307-310.

[8] DAVIES M A, DUTTERER B, PRATT J R, et al. On the dynamics of high-speed milling with long, slender endmills[J]. Annals of the CIRP, 1998, 47(1): 55-60.

[9] ISMAIL F, SOLIMAN E. A new method for the identification of stability lobes in machining $[\mathrm{J}]$. International Journal Machine Tools Manufacture, 1997, 37(6): 763-774.

[10] JENSEN S, SHIN Y. Stability analysis in face milling operations $[\mathrm{J}]$. Journal of Manufacturing Science and Engineering, 1999, 121(4): 600-614.

[11] ALTINTAS Y, ENGIN S, BUDAK E. Analytical stability prediction and design of variable pitch cutters[J]. Transactions of ASME Journal of Manufacturing Science and Engineering, 1999, 121(2): 173-178.

[12] SCHMITZ T L, ZIEGERT J C, STANISLAUS C. A method for predicting chatter stability for systems with speed-dependent spindle dynamics[J]. Aerospace Engineering, 2004, 32: 17-24.

[13] 宋清华. 高速铣削稳定性及加工精度研究[D]. 济南: 山 东大学, 2009.

SONG Qinghua. High-speed milling stability and machining accuracy[D]. Jinan: Shandong University, 2009.

[14] ALTINTAS Y. Manufacturing automation - metal cutting mechanics, machine tool vibrations, and CNC design[M]. Cambridge: Cambridge University, 2002.

[15] 汪博, 孙伟, 闻邦椿. 考虑主轴-刀柄-刀具结合面的高 速主轴系统动力学特性有限元建模 $[\mathrm{J}]$. 机械工程学报, 2012, 48(15): 83-89.

WANG Bo, SUN Wei, WEN Bangchun. The finite 
element modeling of high-speed spindle system dynamics with spindle-holder-tool joints[J]. Journal of Mechanical Engineering, 2012, 48(15): 83-89.

[16] 孙伟, 汪博, 闻邦椿. 高速主轴系统静止及运转状态下 动力学特性对比分析 [J]. 机械工程学报, 2012, 48(11): 146-152.

SUN Wei, WANG Bo, WEN Bangchun. Comparative analysis of dynamics characteristics for static and operation state of high-speed spindle system[J]. Journal of
Mechanical Engineering, 2012, 48(11): 146-152.

作者简介: 汪博(通信作者), 女, 1984 年出生, 师资博士后。主要研究 方向为机床主轴系统动力学和振动测试。

E-mail: wangb@me.neu.edu.cn

孙伟, 男, 1975 年出生, 博士, 副教授。主要研究方向为机械系统动力 学分析及振动控制。

E-mail: weisun@mail.neu.edu.cn

闻邦椿, 男, 1930 年出生, 博士研究生导师, 中国科学院院士。主要研 究方向为转子动力学、非线性动力学和设计方法学。

E-mail: bcwen1930@vip.sina.com 